\title{
Influence of the irradiation dose in the cellular structure of natural rubber foams cross-linked by electron beam irradiation
}

\author{
L.O. Salmazo ${ }^{\mathrm{a}, *}$, A. Lopez-Gil ${ }^{\mathrm{a}, \mathrm{b}}$, Z.M. Ariff ${ }^{\mathrm{d}}$, A.E. Job $^{\mathrm{c}}$, M.A. Rodriguez-Perez ${ }^{\mathrm{a}}$ \\ a Cellular Materials Laboratory (CellMat), Condensed Matter Physics Department, University of Valladolid, Paseo de Belén 7, 47011, Valladolid, Spain \\ b CellMat Technologies S.L. Paseo de Belén 9-A, 47011 Valladolid, Spain \\ ${ }^{c}$ Faculdade de Ciências e Tecnologia, UNESP-Universidade Estadual Paulista, Rua Roberto Simonsen, 305, CEP 19060-900, Presidente Prudente, SP, Brazil \\ d School of Materials and Mineral Resources Engineering, Engineering Campus, Universiti Sains Malaysia, 14300 Nibong Tebal, Pulau Pinang, Malaysia
}

\section{A R T I C L E I N F O}

Article history:

Received 8 February 2016

Received in revised form 6 May 2016

Accepted 11 May 2016

Available online 1 June 2016

\section{Keywords:}

Biopolymer

Dry natural rubber

Electron beam irradiation

Foams

Cellular structure

\begin{abstract}
A B S T R A C T
This work focused on the production of natural rubber foams by cross-linking the polymer matrix using high energy electron beam at different irradiation doses (from 50 to $150 \mathrm{kGy}$ ) and foaming it with a chemical blowing agent. The aim was to study how the irradiation dose influenced the cellular structure morphology of the produced foams. This was accomplished by quantifying the evolution of cellular structure parameters, such as cell size and cell density, with respect to foaming time and irradiation dose. The results showed that the foams irradiated at the lowest dose are more prone to suffer cellular structure degeneration, resulting in the formation of bimodal cellular structures, while foams irradiated with the highest dose, exhibited more uniform cellular structure. In the latter case, the nucleation density clearly increased resulting in the formation of foams with homogeneous cellular structures and smaller cell sizes $(\approx 20 \mu \mathrm{m})$.
\end{abstract}

(c) 2016 Elsevier B.V. All rights reserved.

\section{Introduction}

Natural rubber (NR) is a bioderived elastomeric polymer which is extensively used for the production of tires, gloves and gaskets (Rippel and Bragança, 2009; Kohjiya and Ikeda, 2014). It is harvested from the Hevea Brasiliensis tree as a liquid suspension called latex in which the presence of other substances such as proteins, fatty acids, carbohydrates to name a few, make its properties highly variable. Moreover, latex requires to be chemically stabilized with ammonia prior to being processed because its properties can be environmentally affected during storage. On the contrary, the dried form of NR (i.e. dry natural rubber) which is obtained after a centrifugation process and subsequent drying of latex, present more uniform properties and stability with time (Najib et al., 2011). As a foamed material, NR finds numerous applications especially in household comfort elements such as mattresses, cushions and pillows. However, for these productions, the liquid suspension, latex, is employed as the main raw material (Eaves, 2004). The dried version of NR has not found many application niches as a foamed material so far. In addition, there are few works in literature dealing with the production of NR foams using the dried version of NR

\footnotetext{
* Corresponding author.

E-mail address: lesalmazo@yahoo.com.br (L.O. Salmazo).
}

(Ariff et al., 2008; Joon-Hyung et al., 2006; Joon-Hyung et al., 2007; Najib et al., 2009; Najib et al., 2011).

NR has to be cross-linked during processing in order to have the desired properties not only for being applied as a solid material but also as a foam. For instance, the production of mattresses and pillows (latex foams) is based on the established Talalay and Dunlop processes, which require the cross-linking of the polymer chains (Eaves, 2004). The same is required with the production of automotive tires in which the well-known vulcanization process is used to cross-link the polymer chains (Mark et al., 2005). The NR polymer matrix can be cross-linked either by chemical reagents (peroxides or sulphur) (Banik and Bhowmick, 2000; Manaila et al., 2014) or by irradiation with high energy electron beam. The irradiation process has two clear advantages over the conventional chemical processes: firstly, the final foamed material does not present chemical residues. Secondly, the cross-linking degree can be controlled throughout the applied irradiation dose (ID) before the foaming process. The process has found tremendous interest in the scientific community and there are plenty of articles in the literature regarding the effect of high energy electron radiation not only on the properties of NR, modified NR and their blends (Ahmad et al., 2005; Almaslow et al., 2013; Banik and Bhowmick, 2000; Chirinos et al., 2003; Craciun et al., 2014; Hassan et al., 2007; Jing et al., 2013; Khalid et al., 2010; Manshaie et al., 2011; Mitra et al., 2008; Nabil and Ismail, 2014; Ratnam and Zaman, 1999; 
Ratnam et al., 2000; Sharif et al., 2005; Stelescu et al., 2014) but also on the properties of polyolefins such as low-density polyethylene (LDPE), high-density polyethylene (HDPE) and ethylene-vinyl acetate (EVA) (Eaves, 1988; Gheysari et al., 2001; Gheysari and Behjat, 2001; Khonakdara et al., 2006; Mateev et al., 1996; Mateev and Karageorgiev, 1996).

As far as foaming is concerned, the cross-linking process promotes increment in viscosity and melt strength of the polymer, which provide more stability to the cell wall during cell growth stage of foaming thus giving higher expansion capability with less tendency of cell collapse. It has been reported that the irradiation process is industrially used for the production of flexible closed-cell polyolefin foams (Rodríguez-Pérez, 2005). Moreover, a lot of scientific works have been published in the recent years dealing with the subject (Dias and Silva, 2007; I-Chun et al., 2004; Rezaeiana et al., 2009; Wang et al., 2013; Xing et al., 2008; Youssef et al., 2007) and most of them focused on studying the ID influence towards the obtained cellular structures. In the work of Wang et al. several irradiation doses $(25,50,75$ and $100 \mathrm{kGy})$ were employed for the production of foams based on blends of LDPE/EVA by a physical foaming process where $\mathrm{CO}_{2}$ was used as the blowing agent. Although there were no significant differences between the produced foams in terms of cellular structure, the sample irradiated with $100 \mathrm{kGy}$ showed the lowest average cell size $(\approx 20 \mu \mathrm{m})$. In the work of Youssef et al., a similar study was carried out on LDPE foams with and without blending with EVA. The range of IDs employed was the same (i.e. 25-100 kGy) but in this case, a chemical foaming process was employed in which azodicarbonamide was used as the blowing agent. In this case, there was a drastic reduction of the expansion ratio when the ID was increased. The work of Xing et al. studied the effect of ID (in the same range of the previous works) and the foaming temperature on the expansion ratio and the cellular structure of LDPE foams produced from a gas dissolution foaming process with $\mathrm{CO}_{2}$ as the blowing agent. They discovered that the foaming temperature had more influence in the determination of the final cellular structures than the ID. The only clear trend obtained was that the increment of the ID yielded a reduction of the expansion ratio regardless of the foaming temperature. As far as the authors' knowledge, the only work related to the production of NR foams is the one conducted by Ghazali et al. in which blends of NR/EVA were foamed by a chemical foaming process with different blowing agent contents (Ghazali et al., 1999). These blends were irradiated before foaming with a broader range of IDs than in the previously mentioned works (20-200 kGy). The foams were obtained in a range of densities between 0.3 and $0.5 \mathrm{~g} / \mathrm{cm}^{3}$. The researchers claimed that the increment of the ID restricted the expansion of the polymer. However, the cellular structures were not conveyed and analyzed.

The above review on the existing literature related to the subject has revealed that the control of the cellular structure in terms of cell size and homogeneity by the irradiation process is still a challenge. This work attempted to go further into the subject by analyzing accurately the evolution of cellular structure parameters such as cell size and cell density with respect to the electron beam exposure. The monitoring and evaluation of these parameters will be performed on the produced NR foams after being irradiated with high energy electrons at different doses and foamed via chemical foaming process at different foaming times.

\section{Materials}

Dry natural rubber (NR) of the Crepe Brazilian Clear (CCB) type was employed as the polymer matrix for the production of crosslinked natural rubber foams. Azodicarbonamide (ADC) was used as the blowing agent. It was provided by Lanxess in the form of
Table 1

Characteristics of the reagents and formulations employed.

\begin{tabular}{|c|c|c|c|c|}
\hline Raw material & Chemical formula & $\mathrm{Phr}^{\mathrm{a}}$ & Density $\left(\mathrm{kg} / \mathrm{m}^{3}\right)$ & Purity (\%) \\
\hline Dry natural rubber (NR) & $\left(\mathrm{C}_{5} \mathrm{H}_{8}\right)_{\mathrm{n}}$ & 100 & 930 & - \\
\hline Zinc Oxide & $\mathrm{ZnO}$ & 0.25 & 300 & 94 \\
\hline Azodicarbonamide & $\mathrm{C}_{2} \mathrm{H}_{4} \mathrm{O}_{2} \mathrm{~N}_{4}$ & 10 & 1600 & 99 \\
\hline
\end{tabular}

a Parts per hundred of natural rubber.

yellow powders with an average particle size of $3.9 \mu \mathrm{m}$ and with a decomposition temperature of $210^{\circ} \mathrm{C}$ (data obtained from the technical data sheet). Moreover, Zinc Oxide ( $\mathrm{ZnO})$, which was supplied by Silox, was added to the formulations with the aim of catalyzing the thermal decomposition reaction of the blowing agent. The formulation employed in this study is summarized in Table 1.

\section{Production process}

The production process consisted of four steps: blending, thermoforming, cross-linking and foaming. First, the formulation described in Table 1, was compounded in a Haake internal mixer model Rheodrive 5000. The temperature and the screw speed were set to $60^{\circ} \mathrm{C}$ and $60 \mathrm{rpm}$ respectively. For the second step, the compound was thermoformed in a hot-plate press at a temperature of $80^{\circ} \mathrm{C}$ and by applying a pressure of $10 \mathrm{MPa}$ for $5 \mathrm{~min}$. In this way, solid sheets with $2 \mathrm{~mm}$ in thickness were obtained. In the third step, the obtained solid sheets were subjected to electron beam irradiation in the facilities of MEVION TECHNOLOGY with the aim of cross-linking the polymer matrix. The energy employed was $10 \mathrm{MeV}$, the power of $40 \mathrm{~kW}, 4 \mathrm{~mA}$ intensity and the frequency of $500 \mathrm{~Hz}$. Five solid sheets with different IDs: 50, 75, 100, 125 and $150 \mathrm{kGy}$, were obtained. Finally, small samples $\left(20 \times 20 \mathrm{~mm}^{2}\right)$ extracted from the irradiated natural rubber sheets were foamed by immersing them in a silicon oil bath heated at high temperature $\left(231^{\circ} \mathrm{C}\right)$. Several foams were produced at different times for each irradiation dose: $30,37,45,53,60,75$ and 90 s. All these foams were denominated accordingly throughout the work with the acronym NRF (natural rubber foam) followed by the applied ID. For instance, the natural rubber foam irradiated with $50 \mathrm{kGy}$ was denominated as NRF50. In addition, solid natural rubber sheets were also produced (i.e. without $\mathrm{ADC}$ and $\mathrm{ZnO}$ ) following the same production route. They were subjected to the same IDs previously specified with the aim of evaluating the degree of alteration induced to the virgin polymer matrix by the irradiation process (cross-linking). These samples will be denominated with the acronym NRS (solid natural rubber) followed by the applied ID. NRS50 referred to the sample produced without ADC and irradiated with $50 \mathrm{kGy}$.

\section{Characterization techniques}

\subsection{Density, expansion ratio and porosity measurements}

Density measurements of the foamed samples $\left(\rho_{f}\right)$ were performed by the geometric method; that is by dividing the weight of each specimen between its corresponding volume (ASTM standard D1622-08). The relative density of the foams $\left(\rho_{r}\right)$ was in turn calculated as the ratio between the density of the foam $\left(\rho_{f}\right)$ and that of the corresponding solid $\left(\rho_{s}\right)$ as shown in Eq. (1).

$\rho_{r}=\frac{\rho_{f}}{\rho_{s}}$

The volumetric expansion ratio $(E R)$ of the foams was calculated inversely, that is, as the ratio between the solid density and the foam density (Eq. (2)).

$E R=\frac{\rho_{s}}{\rho_{f}}$ 

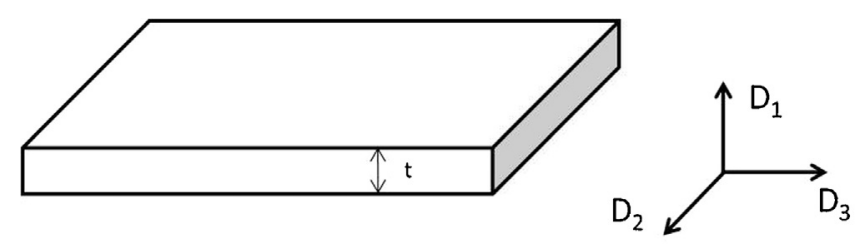

Fig. 1. Scheme of the natural rubber based sheets and indication of their thickness (t).

In addition, the porosity of the foamed samples $\left(V_{f}\right)$, which represents the gas fraction in the material was calculated using Eq. (3).

$V_{f}=1-\rho_{r}$

The density of the solid natural rubber matrix was taken as $930 \mathrm{~kg} / \mathrm{m}^{3}$ in all these calculations.

\subsection{Cellular structure}

The cellular structure of the foamed samples was evaluated by means of micrographs analyses, which were taken by a scanning electron microscope (SEM) model QUANTA 200 FEG. Samples were fractured after being frozen in liquid nitrogen. The fractured surfaces corresponded to one of the planes parallel to the thickness $(t)$ direction $\left(D_{1}\right)$ of the sheets $\left(D_{1} / D_{2}\right.$ or $\left.D_{1} / D_{3}\right)$ as shown in Fig. 1.

The SEM micrographs were taken in these fractured surfaces and they were analyzed by an image analysis software, Image (Pinto et al., 2013) in which a binarization process was performed so as to isolate each cell from the rest allowing the software to measure cellular structure parameters such as the cell size in $2 \mathrm{D}(\phi)$, anisotropy ratio $(R)$, cell density $\left(N_{v}\right)$ and cell nucleation density $\left(N_{0}\right)$. For measuring the size of each cell $\left(\phi_{i}\right)$, the software determined its center and then measured its length $(l)$ in eight different angles. Finally, it calculated the average diameter. The foam cell size $(\phi)$ is the average for all the cells considered in the image as shown in Eq. (4) where $n$ is the total number of cells.

$\phi=\frac{\sum_{i=1}^{n} \phi_{i}}{n}$

The anisotropy ratio $(R)$ was calculated using Eq. (5), which represents the ratio between the cell size of the foam in the direction $\mathrm{D}_{1}\left(\phi_{1}\right)$ and the cell size in one of the directions perpendicular to it $\left(\phi_{2,3}\right)$.

$\boldsymbol{R}=\frac{\phi_{1}}{\phi_{2,3}}$

The cell size distributions were characterized by parameters such as the asymmetry coefficient $(A C)$ and the standard deviation $(S D)$ which accounts for the shape and homogeneity of the distributions, respectively. Both parameters were calculated by Eqs. (6) and (7).

$A C=\frac{\sum_{i}^{n}\left(\phi_{i}-\phi\right)^{3}}{n(S D)^{3}}$

$S D=\frac{\sqrt{\sum_{i}^{n}\left(\phi_{i}-\phi\right)^{2}}}{n-1}$

The homogeneity of the cell size distribution is more accurately described by the NSD (normalized standard deviation) parameter which represents the ratio between the standard deviation and the average cell size of each foam sample (Eq. (8)).

$N S D=\frac{S D}{\phi}$
The cell density or number of cells per cubic centimeter of the foamed material $\left(N_{v}\right)$ was determined by Eq. (9) in which $\mathrm{n}$ is the total number of cells, $\mathrm{M}$ is the magnification of the image and $\mathrm{A}$ is the area considered for the analyses (Kumar, 1988).

$\boldsymbol{N}_{\boldsymbol{v}}=\left[\frac{\left(\boldsymbol{n} \boldsymbol{M}^{2}\right)}{\boldsymbol{A}}\right]^{3 / 2}$

The cell density measurement allows the estimation of the cell nucleation density $\left(N_{0}\right)$ by means of Eq. (10) in which $V_{f}$ represents the porosity of the foams (Eq. (3)).

$N_{0}=\frac{N_{v}}{1-V_{f}}$

\subsection{Cross-linking degree}

The cross-linking degree of the NRS samples were determined by the Flory-Rhener (Eq. (11)), which calculates the density of crosslink points $(v)$ in the irradiated material (Flory and RehnerJr, 1943; Manaila et al., 2014).

$v=-\frac{\ln \left(1-v_{2 m}\right)+v_{2 m}+\chi_{12} v_{2 m}^{2}}{V_{1}\left(v_{2 m}^{1 / 3}-\frac{v_{2 m}}{2}\right)}$

where $\chi_{12}$ is the interaction parameter polymer-solvent (or Flory parameter), which is generally adopted as $0.42 ; V_{1}$ is the solvent molar volume (toluene in this case) which is calculated by Eq. (12) and finally, $v_{2 m}$ is the volume fraction of NR obtained after submerging the sample within the solvent after specific period of time, which was in turn determined through the increase in weight produced after the swelling of the sample (Eq. (13)).

$V_{1}=\frac{M M_{T}}{\rho_{T}}$

$v_{2 m}=\frac{V_{N R}}{V_{T}+V_{N R}}=\frac{\frac{m_{N R}}{\rho_{N R}}}{\frac{m_{T}}{\rho_{T}}+\frac{m_{N R}}{\rho_{N R}}}$

In Eqs. (11) and (12), $M M_{T}$ represents the solvent (toluene) molar mass $(92 \mathrm{~g} / \mathrm{mol}), \rho_{T}$ is the toluene density $\left(0.862 \mathrm{~g} / \mathrm{cm}^{3}\right)$ and $\rho_{N R}$ is the NR density $\left(0.93 \mathrm{~g} / \mathrm{cm}^{3}\right) . V_{N R}, m_{N R}$ and $V_{T}, m_{T}$ are the volume and the mass of NR and the volume and the mass of the solvent, respectively.

\section{Results and discussions}

The cross-linking densities of the irradiated NRS samples are plotted against the irradiation dose as shown in Fig. 2.

The obtained results follow an expected increasing trend with the applied ID. This increment is quite significant between the ID of $100 \mathrm{kGy}$ and $125 \mathrm{kGy}$ and then somehow level off. These results are in agreement with those of previous works, in which the crosslinking degree of NR samples increased with the applied ID and remained practically constant after being irradiated with $100 \mathrm{kGy}$ (Khalid et al., 2010; Manaila et al., 2014; Ratnam et al., 2000; Sharif et al., 2005). Only one of these works employed the FloryRhener method to calculate the cross-linking density of the samples (Manaila et al., 2014) but the absolute values obtained were higher with respect to those obtained in this work. This could be due to the different grade of natural rubber employed.

Fig. 3 shows photographs of the NRF samples produced in this work at the different foaming times.

Some foams, especially those produced at the lower times (30, 37,45 and $53 \mathrm{~s}$.), presented significant distortion. This occurrence was caused by the existence of different blowing agent decomposition rates throughout the sample which was likely caused by 


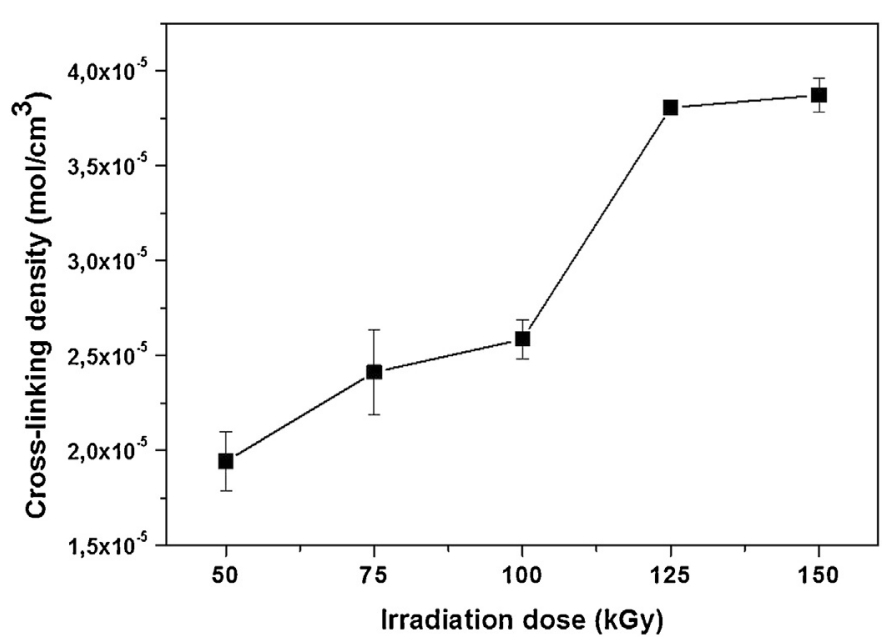

Fig. 2. Crosslinking density versus ID of the NRS samples.

temperature gradient across the samples. Having direct contact to the heating medium, blowing agents within the samples surface started to decompose first but the expansion process in the middle section was somehow delayed due to poor heat transfer of NR. Therefore, when the foaming process was stopped, it was believed that expansion of the precursor in the middle section has not completed yet resulting the final foam to present the observed distortion.

The expansion ratio of all samples seemed to evolve with the foaming time until a certain value where it seems to remain practically constant. In order to quantify this evolution, both the relative density $\left(\rho_{r}\right)$ and the expansion ratio $(E R)$ were represented versus the foaming time as displayed in Fig. 4(a) and (b). The results for the foams produced from the non-irradiated polymer (NRF0) were not included because they presented almost completely collapsed structures, which made it difficult to calculate their geometrical volume.

There is a general trend for all the samples regardless of the ID: $\rho_{r}$ decreased as the foaming time was increased. Consequently, ER followed the opposite tendency; it increased with the increment in foaming time. However, these trends are not linear and the main variations occurred in the range of foaming times between 30 and $53 \mathrm{~s}$. After that, all the foams seem to reach a steady state in which both $\rho_{\mathrm{r}}$ and $E R$ are more or less plateau.

Moreover, it is interesting to note that the stabilized $E R$, is clearly dependent on the applied ID in the sense that the higher the applied ID, the lower the ER. The highest density was obtained in the material irradiated with $150 \mathrm{kGy}\left(\approx 300 \mathrm{~kg} / \mathrm{m}^{3}\right)$ while the lowest density in the material irradiated with $50 \mathrm{kGy}\left(\approx 100 \mathrm{~kg} / \mathrm{m}^{3}\right)$. This behavior remains constant in the range of foaming times of 60-90 s, which could be due to the gradual increase of the cross-linking density obtained after increasing the ID (Fig. 2). On the contrary, when considering lower foaming time, such dependence of the foam density with the ID applied was not found. For instance, at 30 s., the NRF100 foam is the one which presents the lowest $\rho_{r}$. This could be due to the lack of homogeneity of these foams, which made it difficult to get accurate measurements of their volumes. These results are better represented in Fig. 5, in which the ER of all the NRF samples is plotted versus the cross-linking density of the material.

The increment of the cross-linking density can be accompanied by an increment of the viscosity of the polymer matrix. This means that the polymer chains after being cross-linked are less capable of being deformed under the pressure exerted by the gas molecules, which could make the cells to grow slower and give lower expansion rates as reported by several previous works in the literature (Kim and Kim, 2002; Klempner and Frisch, 1991; Sagane and Harayama, 1981; Wang et al., 2013; Youssef et al., 2007).

Having established the foaming behavior of the irradiated NR samples, the study of the cellular structures will be focused on two aspects: firstly, on the influence of the ID. This study was accomplished through observation of SEM micrographs and by measuring the evolution of the cell size $(\phi)$ and the cell density $\left(N_{v}\right)$ with respect to the ID only in the foams produced at $60 \mathrm{~s}$. Secondly, the study focused on evaluating the key mechanisms controlling the evolution of the foaming process, such as nucleation, cell growth and degeneration of the cellular structure. With this purpose in mind, the $\phi$ and $N_{v}$ were measured in foams produced at different foaming times but irradiated at the same exposure levels.

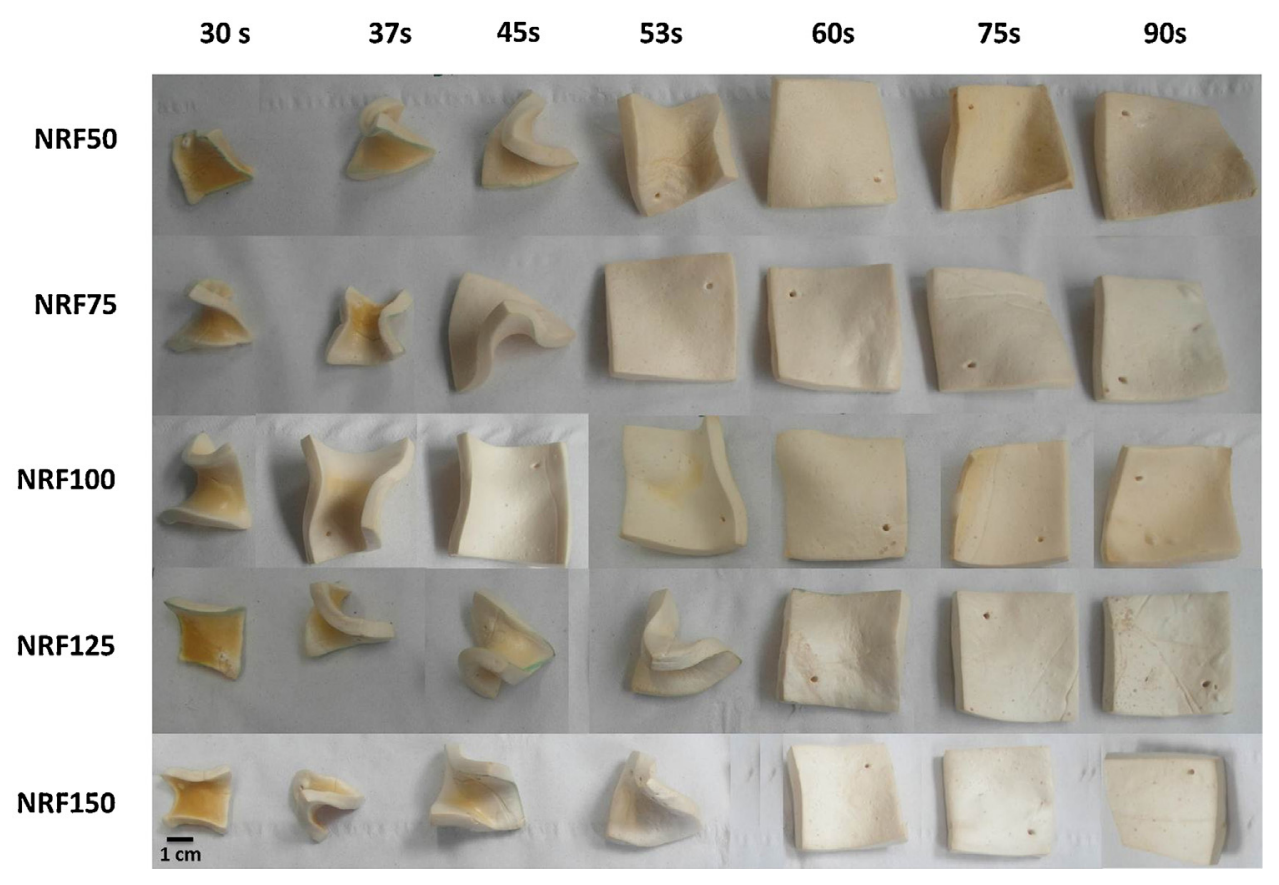

Fig. 3. Pictures of the NRF samples produced. 

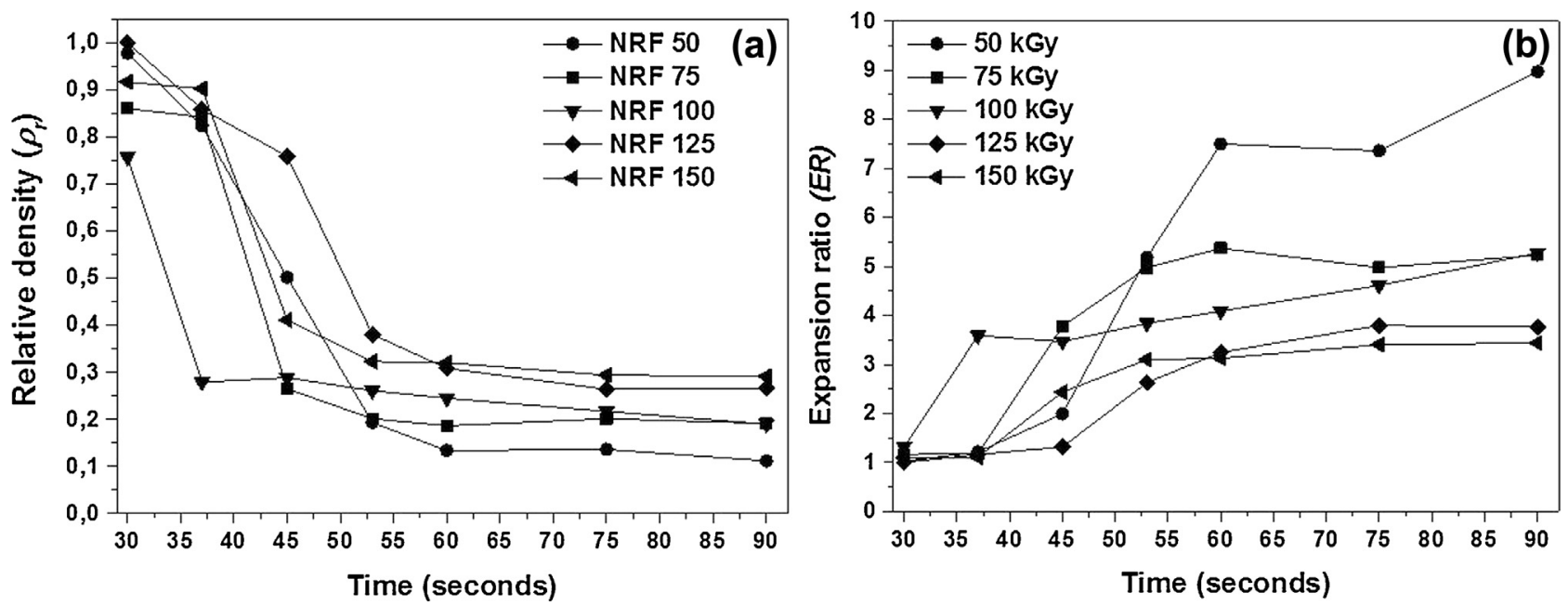

Fig. 4. (a) Relative density ( $\rho r)$ and (b) expansion ratio (ER) of all the NRF samples versus time.

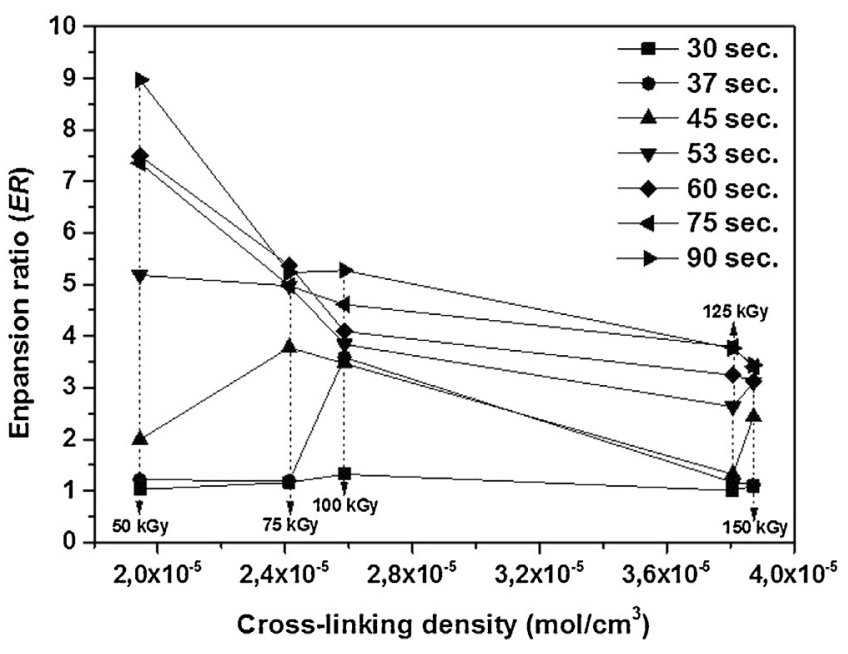

Fig. 5. Expansion ratio versus cross-linking density.

\subsection{Influence of the irradiation dose}

Fig. 6 shows the cellular structure of NR foams irradiated with different doses but produced at the same foaming time ( $60 \mathrm{~s}$.). The cell size distributions ( bin size $=20 \mu \mathrm{m}$ ) corresponding to each foam are included together with the SEM micrographs.

The SEM micrographs show that regardless of the applied ID, all the foams present a closed cellular structure. The increment of ID mainly produced variations in the homogeneity of the cellular structure and cell size. The foams NRF50 and NRF75 presented a characteristic bimodal cell size distribution, which are composed of few large cells surrounded by packs of smaller cells. Fig. 7 shows SEM micrographs of these two foams, which were taken at lower magnifications in order to appreciate in detail the bimodal cellular structures.

These non-homogeneous cellular structures disappeared after gradually increasing the ID applied up to values of 125 and $150 \mathrm{kGy}$. For instance, the sample NRF150 shows a relatively homogeneous cell size distribution in which most of the cells $(>80 \%)$ fall within the range of cell sizes between 20 and $40 \mu \mathrm{m}$. On the contrary, the sample NRF50 shows a wider distribution and a high percentage of the cells (around 35\%) having cell sizes above $100 \mu \mathrm{m}$.

Table 2 shows average parameters of the cellular structures. The ratio cell size $(\phi) /$ expansion ratio (ER) was additionally calculated in order to normalize the effect of the $E R$ on the cell size parameter. Moreover, the relative density $\left(\rho_{r}\right)$ values were also included.

$\phi$ and $N_{v}$ varied significantly with the applied ID but in opposite directions: the general trend is that $\phi$ decreased while $N_{v}$ increased when increasing the ID. There are some exceptions to this general behavior: for instance, the cell size of the sample irradiated with $100 \mathrm{kGy}$ is higher than that of the sample irradiated with $75 \mathrm{kGy}$, but this is likely due to the bimodal cellular structures found when applying low irradiation doses. In these structures there are many small cells whose contribution to the calculus of the average cell size is very significant. Moreover, the average cell size of the sample irradiated with $150 \mathrm{kGy}$ is about $3 \mu \mathrm{m}$ larger than that of the sample irradiated with $125 \mathrm{kGy}$. However, this difference is not very significant, which is in agreement with the fact that the crosslinking densities of both samples are very similar.

The cell density $\left(N_{v}\right)$ of the foam NR150 is 235 times higher than that of the foam NR50. The NSD parameter decreased with the applied ID, which is connected with the fact that the application of higher IDs evolved a more homogeneous cellular structure. The parameter $A C$ also describes this behavior because the lowest value was obtained in the NRF150 foam which possessed the most homogeneous cellular structure from a structural point of view. On the contrary, the anisotropy ratio parameter $(R)$ was not greatly affected by the ID and all the foams presented more or less similar values, around 1 . This is because this structural parameter was controlled by the foaming system rather than by the ID. In this foaming process, the cells were able to grow freely in all directions. Therefore, it was projected that a free foaming process occurred, which usually resulted in the formation of isotropic cells $(R=1)$ as obtained in this work. Similar results to this work were previously reported in other works but they employed different types of polymers: silicon and polyethylene (Liu et al., 2009; Wang et al., 2007).

In principle, these results could be attributed to the fact that the $E R$ of the foams decreased with the applied ID. The $E R$ is a parameter that has been proven to significantly influence the cellular structure of foams (Gibson and Ashby, 1997). Nevertheless, the normalized values of cell size $(\phi / E R)$ also decreased with the increment of the ID. For this reason, such a high variation found in the average cellular structure parameters (Table 2) were more likely to be caused by alteration of the foaming mechanisms (nucleation, expansion and stabilization of the cellular structure), which in turn, could be promoted by alterations of the cross-linking degree of the polymer (Fig. 2). Fig. 8 shows a plot in which both, $\phi$ and $N_{v}$ were represented versus the cross-linking density. 


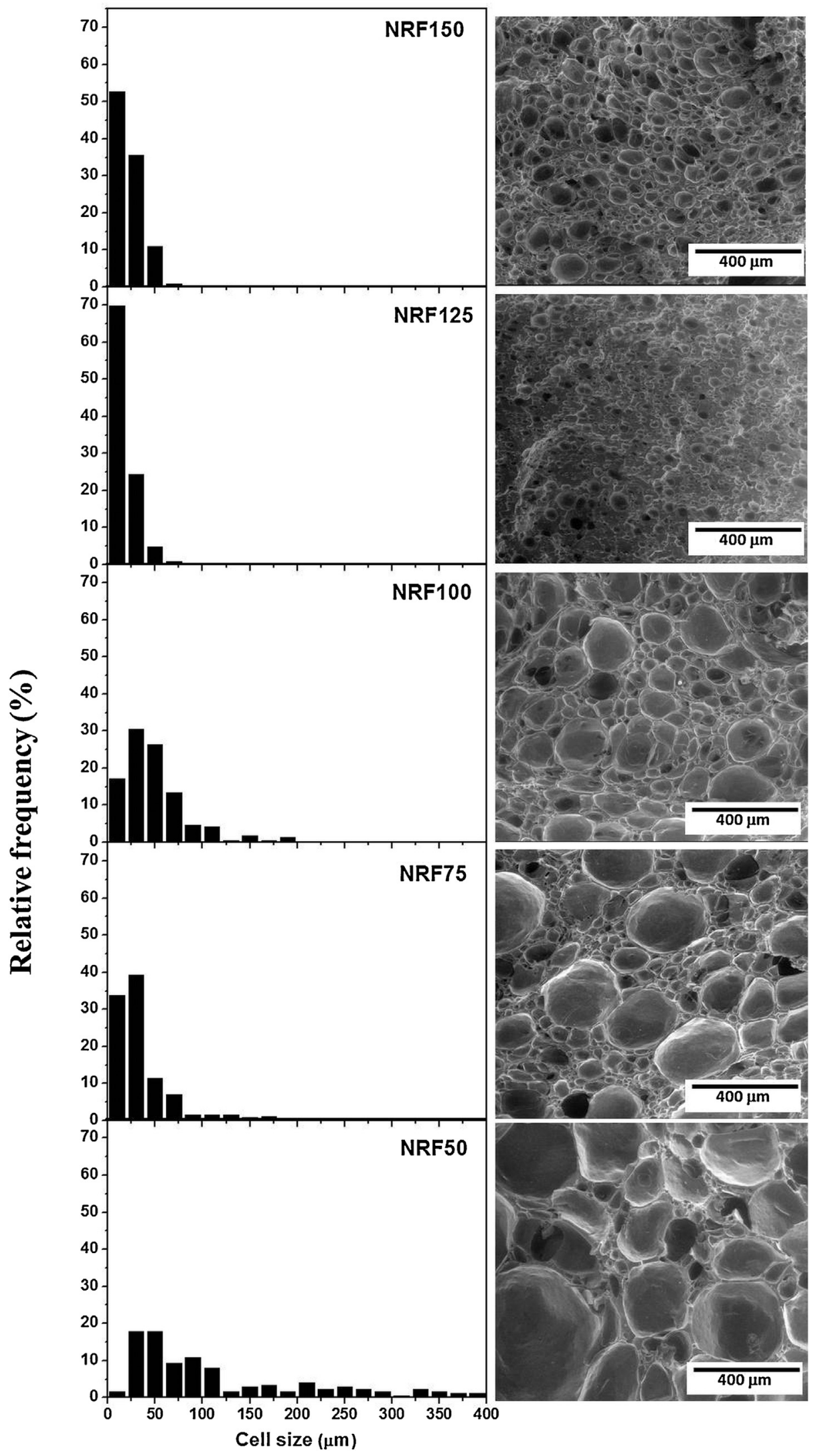

Fig. 6. SEM micrographs of the NR foams produced with different IDs and the same foaming time (60s) and their corresponding cell size distributions.

The production of foams with higher cross-linking densities involved, in general terms, reductions of $\phi$ and increments of $N_{v}$. This chemical alteration of the polymer matrix at different levels by the electron beam irradiation has somehow modified the basic mechanisms involved in the foaming process. These mechanisms are directly connected with the obtained final cellular structures. 


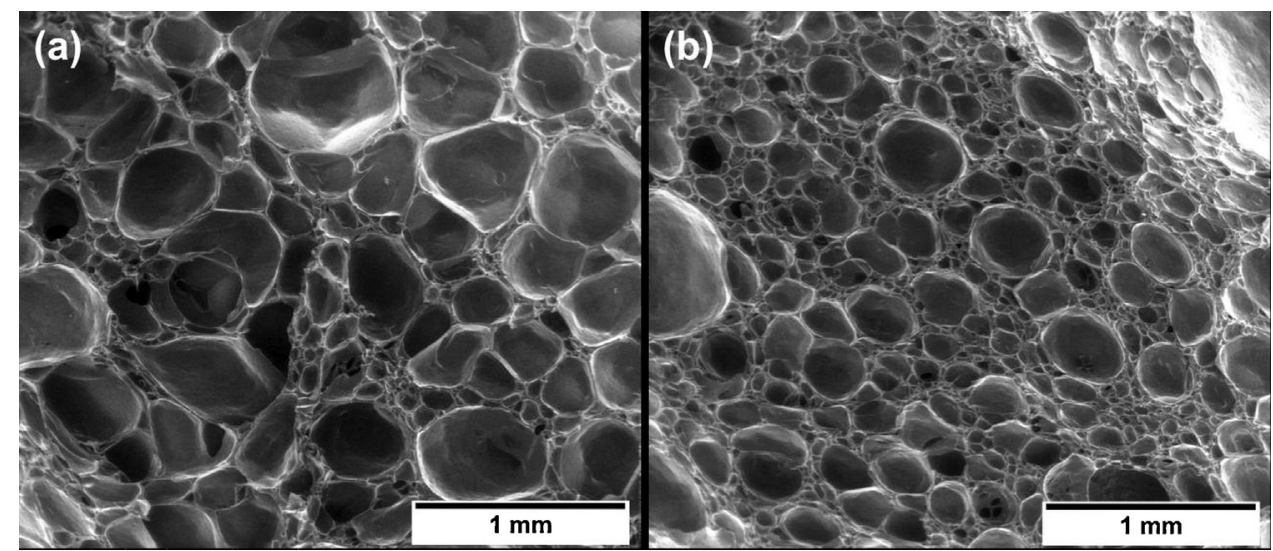

Fig. 7. Bimodal cell size distributions in the (a) NRF50 and (b) NRF75 foams.

Table 2

Average cellular structure parameters and $\rho$ r of the NR foams produced at $60 \mathrm{~s}$.

\begin{tabular}{|c|c|c|c|c|c|}
\hline Irradiation dose (kGy) & 50 & 75 & 100 & 125 & 150 \\
\hline$\rho_{r}$ & 0.13 & 0.18 & 0.23 & 0.30 & 0.30 \\
\hline$\phi(\mu \mathrm{m})$ & 135.0 & 38.0 & 48.8 & 18.0 & 21.6 \\
\hline$\phi E R$ & 17.6 & 6.80 & 11.2 & 5.4 & 6.50 \\
\hline$N_{v} \times 10^{6}\left(\right.$ cells $\left./ \mathrm{cm}^{3}\right)$ & 0.11 & 2.99 & 2.39 & 15.52 & 25.91 \\
\hline$N_{0} \times 10^{5}\left(\right.$ cells $\left./ \mathrm{cm}^{3}\right)$ & 8.70 & 168.1 & 102.4 & 526.9 & 847.3 \\
\hline$N S D$ & 0.95 & 1.03 & 0.70 & 0.63 & 0.60 \\
\hline$A C$ & 1.75 & 3.15 & 1.83 & 1.60 & 0.89 \\
\hline$R$ & 1.10 & 1.10 & 1.00 & 0.90 & 1.00 \\
\hline
\end{tabular}

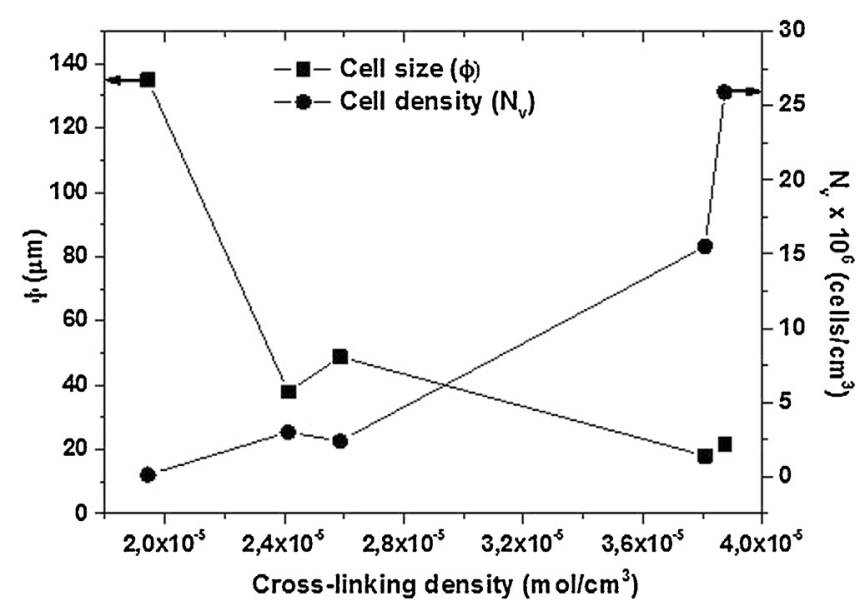

Fig. 8. Cell size $(\phi)$ and Cell density (Nv) versus the cross-linking density.

For instance, an increase of the nucleation rate (higher cell nucleation densities $\left./ N_{0}\right)$ should lead to higher cell densities $\left(N_{v}\right)$ as already observed in previous works (Liu et al., 2009). In the same way, the use of polymers with higher stabilities in the molten state should lead to lower degeneration rates of the cellular structure, as proven by the presence of cells with lower sizes and more homogenous cellular structures.

The two conveyed theories could explain the existence of such variation in cellular structures in this work but with the results obtained so far, it is difficult to corroborate which of them is having more influence. For this reason, the cellular structures of foams from samples irradiated with the lowest and the highest irradiation doses (NR50 and NR150) and produced at different foaming times were also studied. The results obtained are presented in the following section.

\subsection{Influence of the foaming time}

The cellular structures of NR50 and NR150 foams produced at different foaming times (30, 37, 45, 53, 60, 75 and 90 s) are shown sequentially in Fig. 9 together with the corresponding cell size distributions (bin size $=20 \mu \mathrm{m}$ in the case of the NR50 foams and bin size $=10 \mu \mathrm{m}$ in the case of the NR150 foams).

The SEM micrographs allow qualitatively assessment of the cellular structures evolution for both foams produced at different foaming times. It was observed that the cellular structure of the NRF50 sample evolved from non-homogeneous bimodal cell size distributions to clearly degenerated cellular structures with very large cell sizes (75 and 90 s.). On the other hand, the cellular structure of the NRF150 sample do not seem to evolve substantially and they are more homogeneous than those observed in the NRF50 samples in all range of foaming times studied. These observations were also reflected in the quantitative cell size distributions results. Table 2 shows the average parameters calculated from the image analyses of these cellular structures.

The NSD and $A C$ parameters followed very different paths depending on the foams considered. In the case of the NRF50 foams, both values increased with time, which describe perfectly the evolution of the formation of the bimodal cell size distributions. This growing trend of both parameters are hampered at the longest time because the cellular structure is in some way homogenized by cell coalescence mechanisms, which resulted in the formation of larger cells. On the contrary, in the case of the NRF150 foams, the evolutions of these two parameters are practically negligible which indicate higher homogeneity of these cellular structures. The inherent characteristics of the employed foaming process (free foaming) resulted in the formation of highly isotropic cellular structures $(R$ values around 1) as already commented for the foams produced at different IDs (Table 2). Similarly in this case, there is no influence of the applied ID on the cell orientation characteristic.

The results gathered in Table 3 involving $\phi, N_{v}$ and $N_{0}$ proved that these parameters evolved in very different ways depending 

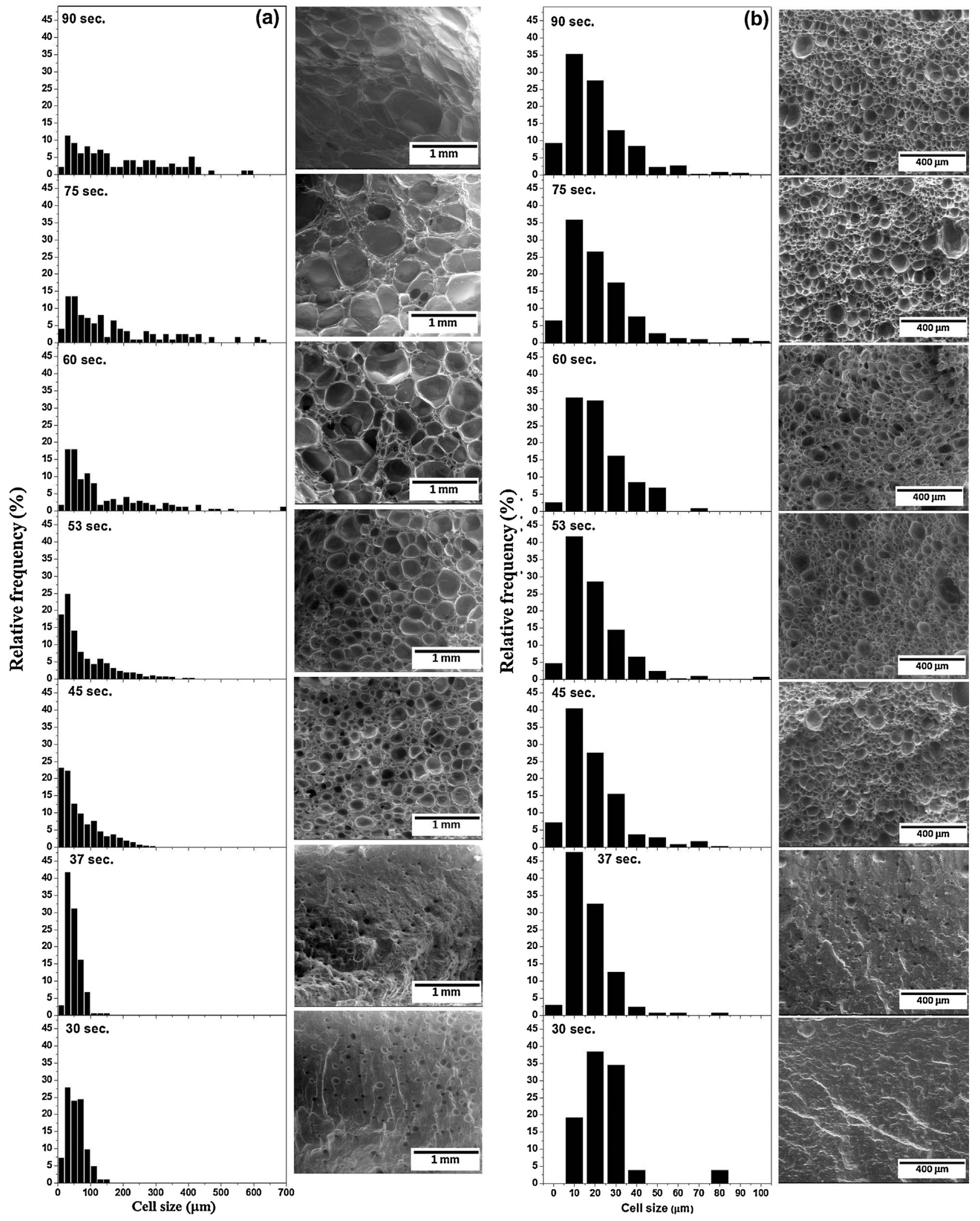

Fig. 9. SEM micrographs of (a) the NRF50 and (b) NRF150 foams produced at different foaming time.

on the applied ID. These two different evolutions are more clearly represented in Figs. 9 and 10 in which both $\phi$ and $N_{0}$, together with the correspondent relative densities $\left(\rho_{r}\right)$, are represented versus the foaming time. For the case of the NRF50 foams (Fig. 10), the obtained values for these three parameters can be split up into two regions: before and after the foaming time of $53 \mathrm{~s}$.

This is because they evolved in different ways before and after this value, which could indicate that the nucleation and degener- 
Table 3

Average cellular structure parameters and $\rho \mathrm{r}$ of the NR50 and NRF150 foams produced at different foaming times.

\begin{tabular}{|c|c|c|c|c|c|c|c|c|}
\hline Foaming time (sec.) & & 30 & 37 & 45 & 53 & 60 & 75 & 90 \\
\hline \multirow[t]{2}{*}{$\rho_{r}$} & NRF50 & 0.94 & 0.79 & 0.48 & 0.18 & 0.13 & 0.13 & 0.11 \\
\hline & NRF150 & 0.92 & 0.90 & 0.41 & 0.58 & 0.30 & 0.29 & 0.29 \\
\hline \multirow[t]{2}{*}{$\phi(\mu \mathrm{m})$} & NRF50 & 55.2 & 47.7 & 68.5 & 79.5 & 135.0 & 165.2 & 189.7 \\
\hline & NRF150 & 25.4 & 16.9 & 19.0 & 19.0 & 21.6 & 21.0 & 20.1 \\
\hline \multirow[t]{2}{*}{$N_{v} \times 10^{6}\left(\right.$ cells $\left./ \mathrm{cm}^{3}\right)$} & NRF50 & 0.14 & 0.12 & 0.49 & 0.44 & 0.11 & 0.06 & 0.04 \\
\hline & NRF150 & 0.10 & 1.59 & 37.75 & 35.47 & 25.91 & 34.68 & 41.13 \\
\hline \multirow[t]{2}{*}{$N_{0} \times 10^{5}\left(\right.$ cells $\left./ \mathrm{cm}^{3}\right)$} & NRF50 & 1.49 & 1.49 & 10.37 & 23.76 & 8.72 & 4.97 & 4.23 \\
\hline & NRF150 & 1.1 & 17.7 & 918.9 & 610.8 & 847.3 & 1181.5 & 1411.4 \\
\hline \multirow[t]{2}{*}{ NSD } & NRF50 & 0.51 & 0.45 & 0.88 & 0.97 & 0.95 & 0.90 & 0.79 \\
\hline & NRF150 & 0.54 & 0.61 & 0.72 & 0.71 & 0.60 & 0.74 & 0.76 \\
\hline \multirow[t]{2}{*}{$A C$} & NRF50 & 0.73 & 1.09 & 1.23 & 1.58 & 1.75 & 1.26 & 1.13 \\
\hline & NRF150 & 2.44 & 2.26 & 1.61 & 2.10 & 0.89 & 1.87 & 1.57 \\
\hline \multirow[t]{2}{*}{$R$} & NRF50 & 0.98 & 0.94 & 0.93 & 1.06 & 1.09 & 0.97 & 0.93 \\
\hline & NRF150 & 0.91 & 0.90 & 1.02 & 0.95 & 1.02 & 0.91 & 1.12 \\
\hline
\end{tabular}

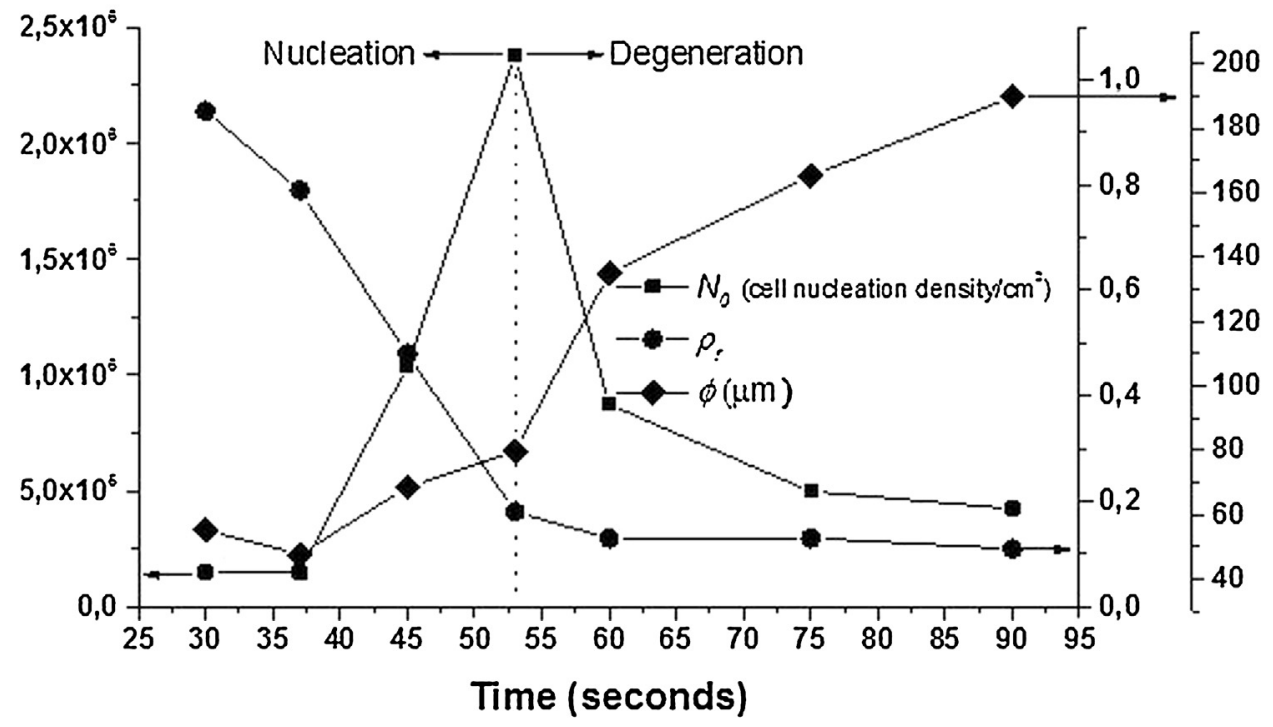

Fig. 10. Evolution of $\phi$, NO and $\rho r$ versus foaming time in the NRF50 foams.

ation mechanisms had different roles in these two regions. The $N_{0}$ values (density of cell nuclei) experienced a clear increment with the foaming time until $53 \mathrm{~s}$. This fact is clearly connected with the blowing agent decomposition reaction, which gradually evolved during this period of time up to its completion (53-60 s.). As ADC is a self-nucleating blowing agent, the higher the amount of the decomposed blowing agent, the higher the number of cell nuclei can be formed (Saiz-Arroyo et al., 2013). In addition, the size of the cells that have already nucleated increases with time due to the increasing amount of gas generated which came from the surrounding polymer matrix to the interior of the cells. This cell size increment can be exclusively associated to cell growth mechanisms as long as the polymer would be strong enough to support the pressure of the gas. At the same time, this fact also caused an increment of the $E R$ of the foam, or inversely, a decrease of $\rho_{\mathrm{r}}$. However, when the foaming time exceeded $53 \mathrm{~s}$, the $N_{0}$ values started to decrease dramatically, while $E R$ remained practically constant. In addition, $\phi$ continued its growing trend. These results could only be explained by considering that the polymer matrix is no longer able to support the pressure of the gas inside the cells and the cellular structure degeneration mechanisms start to prevail over those factors related to nucleation and cell growth.

Results given in Fig. 11 represent the evolution of these parameters in the NRF150 foams.

The $\rho_{r}$ values followed a similar tendency to that obtained with the NR50 foams because a drastic reduction took place at the ini-

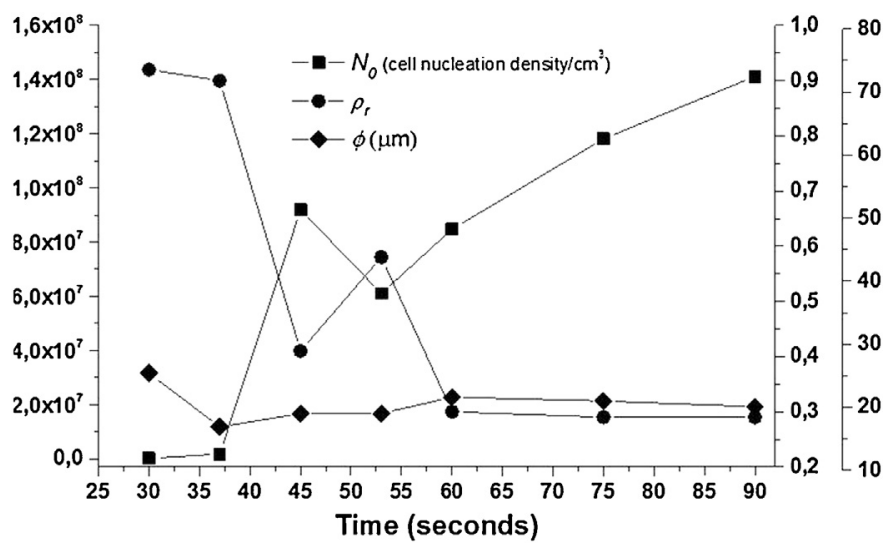

Fig. 11. Evolution of $\phi, N_{0}$ and $\rho_{\mathrm{r}}$ versus foaming time in the NRF150 foams.

tial foaming time while it remained constant during the longer foaming time, i.e. once all the gas has been generated. On the contrary, the trends obtained for $N_{0}$ and $\phi$ are completely different to those obtained with the NR50 foams. Firstly, $N_{0}$ underwent a drastic increment during the initial foaming times. The values achieved after $45 \mathrm{~s}$. are considerably higher than those of the NR50 foams (in some cases up to two orders of magnitude). Since then, they continue to increase but at a lower rate. On the other hand, the 
cell size values obtained (around $20 \mu \mathrm{m}$ ) are very similar for all the considered foaming times (Kumar, 1988).

These trends proved that the degeneration mechanisms did not play a significant role in these foams (NRF150), at least in the range of foaming time studied (30-90 s.). This was due to the highly stable polymer (high cross-linking densities), which was obtained after being irradiated at high IDs (125 and $150 \mathrm{kGy}$ ). The most significant contribution to the formation of these homogeneous cellular structures with very low cell sizes came from the nucleation mechanisms, which were clearly intensified by applying these IDs. This is a phenomenon which has been previously reported in literature (Liu et al., 2009).

\section{Conclusions}

NR foams with low to medium densities $\left(100-300 \mathrm{~kg} / \mathrm{m}^{3}\right)$ and with very low cell sizes $(20 \mu \mathrm{m})$ have been produced by irradiating the polymer matrix with high energy electrons and foaming them through a chemical foaming process. The increment of the applied ID (from 50 to $150 \mathrm{kGy}$ ) yielded a gradual increment of the cross-linking degree, which in turn allowed the production of foams with different expansion ratios and different cellular structures. The foams produced with the lowest IDs presented bimodal cell size distributions with densities around $100 \mathrm{~kg} / \mathrm{m}^{3}$ while the foams produced with the highest IDs presented highly homogeneous cellular structures with very low cell sizes $(20 \mu \mathrm{m})$ and densities around $300 \mathrm{~kg} / \mathrm{m}^{3}$. The analysis of the effect of foaming time has provided more details of the role that the different foaming mechanisms played in samples irradiated with very different doses (50 and $150 \mathrm{kGy}$ ). The foam is more susceptible to suffer cellular structure degeneration after being exposed with low IDs. In fact, it has been possible to detect when these degeneration effects started to dominate exceeding those effects related to nucleation. Therefore, this work has set a protocol that is crucial to be followed in controlling the expansion ratio and cellular structure of natural rubber foams which are produced from NR compounds crosslinked with different doses of electron beam irradiation prior to the foaming process.

\section{Acknowledgments}

This work performed with the financial support from CNPq (Conselho Nacional de Desenvolvimento Científico e Tecnológico-Brasil), MCINN (MAT 2012-34901 and MAT201569234-R), the Junta of Castile and Leon (VA035U13) and FPI grant Ref: BES-2010-038746 (Alberto Lopez-Gil).

\section{References}

Ahmad, A., Mohd, D.H., Abdullah, I., 2005. Electron beam cross-linking of NR/LLDPE blends. Iran. Polym. J. 14, 505-510.

Almaslow, A., Ratnam, C.T., Ghazali, M.J., Talib, R.J., Azhari, C.H., 2013. Effects of electron-beam and sulfur crosslinking of epoxidized natural rubber on the friction performance of semi metallic friction materials. Compos. Part B 54, 377-382.

Ariff, Z.M., Zakaria, Z., Tay, L.H., Lee, S.Y., 2008. Effect of foaming temperature and rubber grades on properties of natural rubber foams. J. Appl. Polym. Sci. 107, 2531-2538, http://dx.doi.org/10.1002/app.27375.

Banik, I., Bhowmick, A.K., 2000. Effect of electron beam irradiation on the properties of crosslinked rubbers. Radiat. Phys. Chem. 58, 293-298.

Chirinos, H., Yoshii, F., Makuuchi, K., Lugao, A., 2003. Radiation vulcanization of natural rubber latex using $250 \mathrm{keV}$ electron beam machine. Nucl. Instrum. Meth B 208, 256-259.

Craciun, M.G., Stelescu, M.D., Ighigeanu, D., Ficai, M., 2014. Radiation vulcanization of natural rubber with polyfunctional monomers. Polym. Bull. 71, 57-58.

Dias, D.B., Silva, L.G.A., 2007. Polyethylene foams cross-linked by electron beam. Radiat. Phys. Chem. 76, 1696-1697.

Eaves, D.E., 1988. Ethylene homo and copolymer foams. Cell. Polym. 7, 297.

Eaves, D. (Ed.), 2004. Handbook of Polymer Foams. Rapra Technology, Shrewsbury.

Flory, P.J., RehnerJr, J., 1943. Statistical mechanics of cross linked polymer networks II. Swelling J. Chem. Phys. 11, 521-526.
Ghazali, Z., Johnson, A.F., Dahlan, K.Z., 1999. Radiation crosslinked thermoplastics natural rubber (TPNR) foams. Radiat. Phys. Chem. 55, 73-79.

Gheysari, D., Behjat, A., 2001. Radiation crosslinking of LDPE and HDPE with 5 and $10 \mathrm{MeV}$ electron beams. Eur. Polym. J. 37, 2011-2016.

Gheysari, D., Behjat, A., Haji-Saei, M., 2001. The efect of high-energy electron beam on the mechanical and thermal properties of LDPE and HDPE. Eur. Polym. J. 37, 295-302.

Gibson, L.J., Ashby, M.F., 1997. Cellular Solids: Structure and Properties, 2nd ed. Cambridge University Press, Cambridge.

Hassan, M.M., Mahmoud, G.A., El-Nahas, H.H., Hegazy El-Sayed, A., 2007. Reinforced material from reclaimed rubber/natural rubber, using electron beam and thermal treatment. J. Appl. Polym. Sci. 104, 2569-2578.

I-Chun, Chih-Kuang, L., Tsiang, C., Chien-Chao, R., 2004. Foaming of electron-beam irradiated LDPE blends containing recycled polyethylene foam. J. Polym. Res. $11,149-159$.

Jing, S., Shipeng, W., Yishi, D., Ning, L., Liqun, Z., Yusheng, Y., Li, L., 2013. The network and properties of the NR/SBR vulcanizate modified by electron beam irradiation. Radiat. Phys. Chem. 92, 99-104.

Joon-Hyung, K., Kyo-Chang, C., Jin-Min, Y., 2006. The foaming characteristics and physical properties of natural rubber foams: effects of carbon black content and foaming pressure. J. Ind. Eng. Chem. 12, 795-801.

Joon-Hyung, K., Jae-Song, K., Kyo-Chang, C., Jin-Min, Y., Soo-Yeon, K., 2007. Effects of foaming temperature and carbon black content on the cure characteristics and mechanical properties of natural rubber foams. J. Ind. Eng. Chem. 13, 198-205.

Khalid, M., Ismail, A.F., Ratnam, C.T., Faridah, Y., Rashmi, W., Khatib, M.F.A., 2010 Effect of radiation dose on the properties of natural rubber nanocomposite. Radiat. Phys. Chem. 79, 1279-1285.

Khonakdara, H.A., Jafarib, S.H., Wagenknechtc, U., Jehnichenc, D., 2006. Effect of electron-irradiation on cross-link density and crystalline structure of low- and high-density polyethylene. Radiat. Phys. Chem. 75, 78-86.

Kim, D.W., Kim, K.S., 2002. Electron beam irradiation of noncrosslinked LDPE-EVA foam. J. Cell. Plast. 38, 471-496.

Klempner, D., Frisch, K.C., 1991. Handbook of Polymeric Foams and Foam Technology, 2nd ed. Bergisch Gladbach, Germany.

Kohjiya, S., Ikeda, Y., 2014. Chemistry, Manufacture and Applications of Natural Rubber, 1st ed. Kyoto Institute of Technology, Japan.

Kumar, V., 1988. Process Synthesis for Manufacturing Microcellular Thermoplastic Parts PhD Thesis. Massachusetts Institute of Technology, Cambridge, MA

Liu, P., Liu, D., Zou, H., Fan, P., Xu, W., 2009. Structure and properties of closed-Cell foam prepared from irradiation crosslinked silicone rubber. J. Appl. Polym. Sci. 113, 3590-3595.

Manaila, E., Craciun, G., Stelescu, M.D., Ighigeanu, D., Ficai, M., 2014. Radiation vulcanization of natural rubber with polyfunctional monomers. Polym. Bull. 71, 57-82.

Manshaie, R., Khorasani, S.N., Veshare, S.J., Abadchi, M.R., 2011. Effect of on the properties of natural rubber (NR)/styrene-butadiene rubber (SBR) blend. Radiat. Phys. Chem. 80, 100-106.

Mark, J.E., Erman, B., Eirich, F.R., 2005. The Science and Technology of Rubber, 3rd ed. Academic Press, Washington, DC, USA.

Mateev, M., Karageorgiev, S.S., 1996. Electron Microscopy Analyses of the effect of electron beam irradiation on the macromolecular structure and crosslinking parameters in low density polyethylene films. Radiat. Phys. Chem. 48, 443-448.

Mateev, M., Karageorgiev, S., Atanasonova, B., 1996. Gel-Sol Analysis of the effect of electron beam irradiationon the macromolecular structure and crosslinking parameters in low density polyethylene films. Radiat. Phys. Chem. 48, 437-442.

Mitra, S., Chattopadhyay, S., Bharadwaj, Y.K., Sabharwal, S., Bhowmick, A.K., 2008. Effect of electron beam-cross-linked gels on the rheological properties of raw natural rubber. Radiat. Phys. Chem. 77, 630-642.

Nabil, H., Ismail, H., 2014. Enhancing the thermal stability of natural rubber/recycled ethylene-propylene-diene rubber blends by means of introducing pre-vulcanised ethylene-propylene-diene rubber and electron beam irradiation. Mater. Design 56, 1057-1067.

Najib, N.N., Ariff, Z.M., Manan, N.A., Bakar, A.A., Sipaut, C.S., 2009. Effect of blowing agent concentration on cell morphology and impact properties of natural rubber foam. J. Phys. Sci. 20, 13-25, 10.1.1.516.4653.

Najib, N.N., Ariff, Z.M., Bakar, A.A., Sipaut, C.S., 2011. Correlation between the acoustic and dynamic mechanical properties of natural rubber foam: effect of foaming temperature. Mater. Design 32 (505-511), 19, http://dx.doi.org/10. 1016/j.matdes.2010.08.030.

Pinto, J., Solórzano, E., Rodriguez-Perez, M.A., De Saja, J.A., 2013. Characterization of the cellular structure based on user-interactive image analyses procedures. J. Cell. Plast. 49, 555-575.

Ratnam, C.T., Zaman, K., 1999. Modification of PVC/ENR blend by electron beam irradiation: effect of crosslinking agents. Nucl. Instrum. Meth B 152, 335-34.

Ratnam, C.T., Nasir, M., Baharin, A., Zaman, K., 2000. Electron beam irradiation of epoxidized natural rubber. Nucl. Instrum. Meth B. 171, 455-464.

Rezaeiana, I., Jafaria, S.H., Zahedia, P., Ghaffarib, M., Afradian, S., 2009. Improvements of physical and mechanical properties of electron beam irradiation-crosslinked EVA foams. Polym. Adv. Technol. 20, 487-492.

Rippel, M.M.I., Bragança, F.C., 2009. Borracha natural e nanocompósitos com argila. Quim. Nova 32, 818-882.

Rodríguez-Pérez, M.A., 2005. Crosslinked polyolefin foams production, structure properties, and applications. Adv. Polym. Sci. 184, 97-126.

Sagane, N., Harayama, H., 1981. Plastic foam-radiation cross-linked polyethylene foam. Radiat. Phys. Chem. 18, 19-108. 
Saiz-Arroyo, C., Rodriguez-Perez, M.A., Tirado, J., Lopez-Gil, A., De Saja, J.A., 2013. Structure-property relationships of medium-density polypropylene foams. Polym. Int. 62, 1324-1333.

Sharif, J., Yunus, M.Z.W., Hj, K.Z., Dahlan, M., Ahmad, M.H., 2005. Preparation and properties of radiation crosslinked natural rubber/clay nanocomposites. Polym. Test. 24, 211-217.

Stelescu, M.D., Manaila, E., Craciun, G., Dumitrascu, M., 2014. New green polymeric composites based on hemp and natural rubber processed by electron beam irradiation. Sci. World J. 2014, 1-13.

Wang, B., Wang, M., Xing, Z., Zeng, H., Wu, G., 2007. Characterization of LDPE and LDPE/EVA blends crosslinked by electron beam irradiation and foamed with chemical foaming agent. J. Polym. Res. 14, 351-357.
Wang, B., Wang, M., Xing, Z., Zeng, H., Wu, G., 2013. Preparation of radiation crosslinked foams from low-density polyethylene/ethylene-vinyl acetate (LDPE/EVA) copolymer blend with a supercritical carbon dioxide approach. J. Appl. Polym. Sci. 127, 912-918.

Xing, Z., Wu, G., Huang, S., Chen, S., Zeng, H., 2008. Preparation of microcellular cross-linked polyethylene foams by a radiation and supercritical carbon dioxide approach. J. Supercrit. Fluid 47, 281-289.

Youssef, H.A., Senna, M.M., Eyssa, H.M., 2007. Characterization of LDPE and LDPE/EVA blends crosslinked by electron beam irradiation and foamed with chemical foaming agent. J. Polym. Res. 14, 351-357. 